\title{
New medical marijuana regulations: the coming storm
}

\author{
Meldon Kahan MD, Anita Srivastava MD
}

See related commentary on page 897 and at www.cmaj.ca/lookup/doi/10.1503/cmaj.140309

$\mathrm{H}$ ealth Canada's Marijuana for Medical Purposes Regulations took effect Apr. 1, 2014. Patients are now able to purchase dried cannabis from a licensed distributer if they have a physician's prescription specifying the daily dose and monthly quantity. Although cannabis may be prescribed for any condition, we have focused our comments on chronic pain, which is the most common reason for medical marijuana use.

The Canadian Medical Association, the College of Family Physicians of Canada and the Federation of Medical Regulatory Authorities of Canada have all opposed the new regulations because of the lack of evidence on the safety and efficacy of smoked cannabis. Their concerns are justified: only five randomized controlled trials of smoked cannabis have been conducted, ranging in duration from 1 to 15 days, with a combined total of 182 participants. ${ }^{1}$ Smoked cannabis was compared with placebo rather than other treatment modalities. The trials found that it was superior to placebo in the management of neuropathic pain associated with HIV infection, multiple sclerosis and surgery.'

Smoked cannabis has short- and long-term safety risks. Smoking is an uncontrolled delivery system that rapidly produces high plasma levels of delta-9-tetrahydrocannabinol (THC), the primary psychoactive ingredient of cannabis. A systematic review of controlled trials of cannabis preparations, including synthetic oral formulations, reported increased rates of altered perception, motor function and cognition, ${ }^{2}$ which could cause functional impairment with long-term use. Smoking cannabis has been associated with an increased risk of motor vehicle crashes, schizophrenia, mood disorders and addiction. ${ }^{3,4}$ Observational studies have shown that the risk of these outcomes is higher among those under 25 years old and those who have a current or past history of psychosis or substance use disorder. ${ }^{3}$

Moreover, combustion of dried cannabis produces hundreds of toxic products, some of which are carcinogenic. Although previous studies have had conflicting results, a recent large, long-term retrospective cohort study has shown that smoking cannabis is associated with an increased risk of lung cancer. ${ }^{5}$ There have been case reports of younger adults experiencing acute coronary events shortly after smoking cannabis. ${ }^{6}$ Vaporizing produces much lower concentrations of exhaled carbon monoxide than smoking, but smoking remains the most popular delivery route.

Canadian physicians will be asked by patients to prescribe dried cannabis for chronic pain, despite its safety risks and weak evidence of efficacy. We therefore urgently need prescribing guidelines to help us understand the evidence, to counsel our patients and to know when and how to prescribe cannabis.

The guidelines will need to define the indications, precautions and contraindications for smoked cannabis. Based on the limited controlled trials, the only clear indications for its use are neuropathic pain conditions and spasticity associated with multiple sclerosis. ${ }^{1}$ It might also be considered for palliative care and for severe biomedical pain conditions such as rheumatoid arthritis. ${ }^{1}$ In all cases, smoked cannabis should be reserved for
Competing interests: The authors have received honoraria from Reckitt Benckiser, makers of Suboxone (buprenorphinenaloxone).

This article has been peer reviewed.

Correspondence to:

Meldon Kahan,

meldon.kahan@wchospital.ca

CMAJ 2014. DOI:10.1503 /cmaj.131821 
patients whose pain does not respond to standard analgesics or pharmaceutical cannabinoids. Two pharmaceutical cannabinoids available in Canada are nabilone, an oral product, and nabixamols, an oral-buccal preparation. Both are safer and have greater evidence of efficacy than smoked cannabis. For example, in an experimental study, dronabinol (an oral cannabinoid not available in Canada) had an equal intensity and longer duration of analgesia than smoked cannabis for cold-induced pain in healthy volunteers. ${ }^{7}$ Contraindications to and precautions for smoked cannabis would likely include young age, a history of psychosis or substance abuse, and a history of poorly controlled mood or anxiety disorder.

These indications and contraindications will exclude many people who are current medical marijuana users. Most of them have common pain conditions such as fibromyalgia and back pain, ${ }^{8}$ for which there is little evidence of benefit from smoked cannabis. One study found that patients with fibromyalgia who used medical marijuana were more likely than others with fibromyalgia to have current unstable mental illness and substance use disorders. ${ }^{9}$

The guidelines will also need to recommend a dose that has been shown to be effective for neuropathic pain but does not cause cannabis intoxication. One controlled trial found that $25 \mathrm{mg}$ (roughly equivalent to one inhalation) of $9 \%$ THC relieved neuropathic pain and caused minimal intoxication. ${ }^{1,10}$ The duration of analgesic action of smoked cannabis is probably about three to four hours. In our view, the maximum safe dose, therefore, would be about one inhalation of $9 \%$ THC four times daily, or $400 \mathrm{mg}$ of dried cannabis per day. (Health Canada allows prescriptions of up to $5 \mathrm{~g} / \mathrm{d}$.) Physicians should specify on the prescription that the THC concentration not exceed 9\%. Licensed producers are marketing strains containing $15 \%-25 \%$ THC or higher. Such potent strains are neither necessary nor safe; acute cognitive effects of cannabis are related to the THC dose.

Patients who request cannabis should be assessed for problematic use of cannabis and other substances. Cannabis use disorder should be suspected in patients who are heavy daily smokers, who spend large amounts of time and money on smoking, and who have impaired work or school performance or dysfunctional social relationships. Other features of cannabis use disorder include repeated attempts to quit or reduce use, and risk factors (young age, concurrent anxiety or mood disorder and misuse of other substances).
Patients who use cannabis recreationally should be advised of strategies to reduce exposure to cannabis smoke and to reduce the harms of cannabis intoxication. ${ }^{3}$ Strategies include not smoking daily, not holding one's breath when inhaling, using a vaporizer rather than smoking, not adding tobacco to cannabis cigarettes, not mixing cannabis with alcohol or sedating drugs and not driving for at least six hours after use. Highrisk groups, such as adolescents and patients with substance use or psychiatric disorders, should be advised to smoke only occasionally or not at all.

Some current medical marijuana smokers will be dissatisfied with the physician's decision not to prescribe cannabis if it is contraindicated or not indicated. The best strategy for dealing with patient disagreements is to stick to unambiguous and honest messaging: "I am not comfortable prescribing smoked cannabis because it has little evidence of efficacy for your condition and considerable evidence of harm." Physicians should advise patients with a suspected cannabis use disorder to abstain from cannabis and should refer them for treatment.

\section{References}

1. Controlled Substances and Tobacco Directorate. Information for health care professionals: cannabis (marihuana, marijuana) and the cannabinoids. Ottawa (ON): Health Canada; 2013

2. Martín-Sánchez E, Furukawa TA, Taylor J. et al. Systematic review and meta-analysis of cannabis treatment for chronic pain. Pain Med 2009;10:1353-68.

3. Fischer B, Jeffries V, Hall W. et al. Lower risk cannabis use guidelines for Canada (LRCUG): a narrative review of evidence and recommendations. Can J Public Health 2011;102:324-7.

4. Kalant H. Adverse effects of cannabis on health: an update of the literature since 1996. Prog Neuropsychopharmacol Biol Psychiatry 2004;28:849-63.

5. Callaghan RC, Allebeck P, Sidorchuk A. Marijuana use and risk of lung cancer: a 40-year cohort study. Cancer Causes Control 2013;24:1811-20.

6. Singla S, Sachdeva R, Mehta J L. Cannabinoids and atherosclerotic coronary heart disease. Clin Cardiol 2012;35:329-35.

7. Cooper ZD, Comer SD, Haney M. Comparison of the analgesic effects of dronabinol and smoked marijuana in daily marijuana smokers. Neuropsychopharmacology 2013;38:1984-92

8. Aggarwal SK, Carter GT, Sullivan MD, et al. Characteristics of patients with chronic pain accessing treatment with medical cannabis in Washington State. J Opioid Manag 2009:5:257-86.

9. Ste-Marie PA, Fitzcharles MA, Gamsa A, et al. Association of herbal cannabis use with negative psychosocial parameters in patients with fibromyalgia. Arthritis Care Res (Hoboken) 2012; 64:1202-8.

10. Ware MA, Wang T, Shapiro S, et al. Smoked cannabis for chronic neuropathic pain: a randomized controlled trial. CMAJ 2010; 182:E694-701.

Affiliations: Department of Family Medicine (Kahan), University of Toronto; Substance Use Service (Kahan), Women's College Hospital; Department of Family and Community Medicine (Srivastava), University of Toronto; Urban Family Health Team (Srivastava), St. Joseph's Health Centre, Toronto, Ont.

Contributors: Both authors contributed equally to the conception, writing and revising of the manuscript. They both approved the final version submitted for publication and have agreed to act as guarantors of the work. 